\title{
Classes and Inheritance in Actor-Oriented Design
}

\author{
EDWARD A. LEE \\ UC Berkeley \\ and \\ XIAOJUN LIU \\ Sun Microsystems \\ and \\ STEPHEN NEUENDORFFER \\ Xilinx Research Labs
}

Actor-oriented components emphasize concurrency and temporal semantics and are used for modeling and designing embedded software and hardware. Actors interact with one another through ports via a messaging schema that can follow any of several concurrent semantics. Domainspecific actor-oriented languages and frameworks are common (Simulink, LabVIEW, SystemC, etc.). However, they lack many modularity and abstraction mechanisms that programmers have become accustomed to in object-oriented components, such as classes, inheritance, interfaces, and polymorphism, except as inherited from the host language. This paper shows a form that such mechanisms can take in actor-oriented components, gives a formal structure, and describes a prototype implementation. The mechanisms support actor-oriented class definitions, subclassing, inheritance, and overriding. The formal structure imposes structural constraints on a model (mainly the "derivation invariant") that lead to a policy to govern inheritance. In particular, the structural constraints permit a disciplined form of multiple inheritance with unambiguous inheritance and overriding behavior. The policy is based formally on a generalized ultrametric space with some remarkable properties. In this space, inheritance is favored when actors are "closer" (in the generalized ultrametric), and we show that when inheritance can occur from multiple sources, one source is always unambiguously closer than the other.

Categories and Subject Descriptors: D.3.3 [Programming Languages]: Language Constructs and Features - data types and structures

General Terms: Embedded Systems, Actor-Oriented Design

Additional Key Words and Phrases: actors, components, generalized ultrametric, inheritance, interfaces, overriding, type systems

This paper describes work that is part of the Ptolemy project, which is supported by the National Science Foundation (NSF award number CCR-00225610), and Chess (the Center for Hybrid and Embedded Software Systems), which receives support from NSF, the State of California Micro Program, and the following companies: Agilent, Bosch, DGIST, Hewlett Packard, Microsoft, National Instruments, and Toyota. Additional support is provided by the Army Research Laboratory (Cooperative Agreement Number W911NF-07-2-0019). The views and conclusions contained in this document are those of the authors and should not be interpreted as representing the official polices, either expressed or implied, of the Army Research Laboratory or the U.S. Government. The U.S. Government is authorized to reproduce and distribute reprints for Government purposes notwithstanding any copyright notation hereon.

Permission to make digital/hard copy of all or part of this material without fee for personal or classroom use provided that the copies are not made or distributed for profit or commercial advantage, the ACM copyright/server notice, the title of the publication, and its date appear, and notice is given that copying is by permission of the ACM, Inc. To copy otherwise, to republish, to post on servers, or to redistribute to lists requires prior specific permission and/or a fee.

(C) $20 \mathrm{YY}$ ACM 0000-0000/20YY/0000-0001 $\$ 5.00$ 


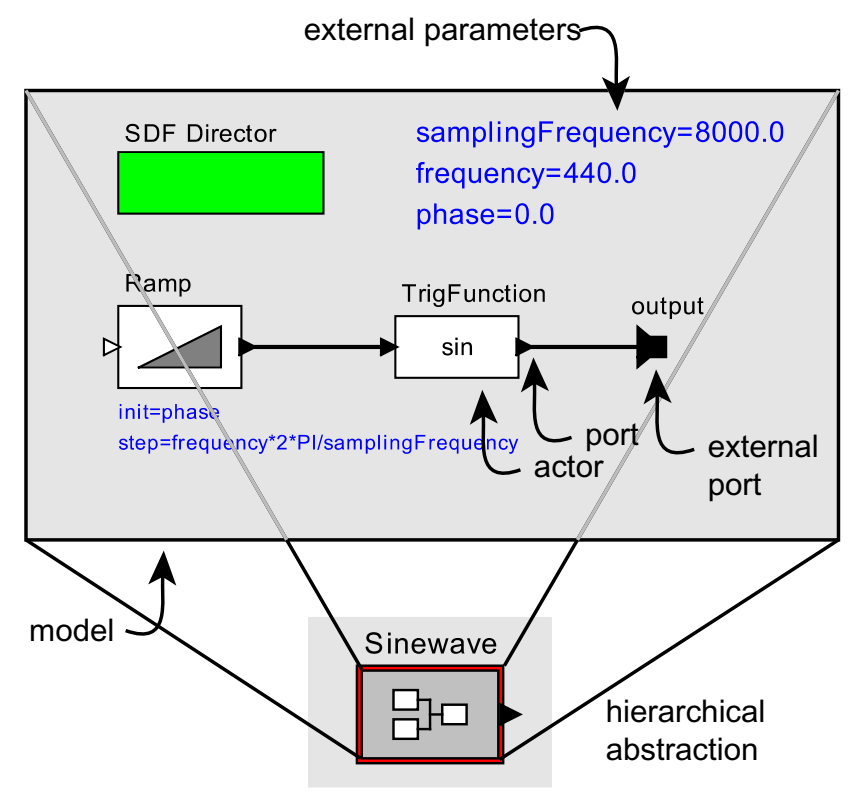

Fig. 1. Illustration of an actor-oriented model (above) and its hierarchical abstraction (below).

\section{INTRODUCTION}

Actor-oriented design is a component methodology that has proven effective for embedded software, hardware design, and domain-specific modeling. Components that we call actors execute and communicate with other actors in a model, as illustrated in figure 1. Actors have a well defined component interface. This interface abstracts the internal state and behavior of an actor, and restricts how an actor interacts with its environment. The interface includes ports that represent points of communication for an actor, and parameters which are used to configure the operation of an actor. Often, parameter values are part of the a priori configuration of an actor and do not change when a model is executed. The configuration of a model also contains explicit communication channels that pass data from one port to another. The use of channels to mediate communication implies that actors interact only with the channels that they are connected to and not directly with other actors.

Like actors, which have a well-defined external interface, a model may also define an external interface, which we call its hierarchical abstraction. This interface consists of external ports and external parameters, which are distinct from the ports and parameters of the individual actors in the model. The external ports of a model can be connected by channels to other external ports of the model or to the ports of actors that compose the model. External parameters of a model can be used to determine the values of the parameters of actors inside the model. A model, therefore, is an actor.

Taken together, the concepts of models, actors, ports, parameters and channels describe the abstract syntax of actor-oriented design (this has also been called

ACM Journal Name, Vol. V, No. N, Month 20YY. 
"static semantics" [Ledeczi et al. 2001] and "structural semantics" [Jackson and Sztipanovits 2006]). This syntax can be represented concretely in several ways, such as graphically, as in figure 1, in XML [Lee and Neuendorffer 2000], in a program designed to a specific object-oriented API, as in SystemC, which has roots in Scenic [Liao et al. 1997], or in an actor-oriented language, such as StreamIT [Thies et al. 2002] or Cal [Eker and Janneck 2003]. Ptolemy II [Eker et al. 2003] offers all four alternatives.

The focus on structured concurrency in actor-oriented (AO) design easily falls within early conceptions of object-oriented (OO) design. But in the last two decades or so, OO design has crystalized into a style, represented by Java, C\#, C++, and UML, that distinctly lacks structured mechanisms for managing concurrency [Johnson 1994]. So-called "active objects" are a step in the right direction, but they are little more than disguised threads, and offer little help to the designer. Threads are a notoriously poor concurrency model for reliable systems [Lee 2006]. Our objectives in this paper are to offer a component technology that brings all the benefits of $\mathrm{OO}$ design but with much better concurrency models.

Actor-oriented design has been around since at least 1966, when Bert Sutherland used one of the first acknowledged object-oriented frameworks, Sketchpad [Sutherland 1963], created by his brother Ivan Sutherland, to build the first actor-oriented programming language (which had a visual syntax) [Sutherland 1966]. Today, actor-oriented languages and frameworks often have visual syntaxes (e.g. Simulink and LabVIEW), and are frequently built on top of object-oriented languages in order to leverage their modularity mechanisms [Buck et al. 1994].

$\mathrm{AO}$ languages, like $\mathrm{OO}$ languages, emphasize modularity of software. In $\mathrm{AO}$ design, components are concurrent objects that communicate via messaging, as opposed to abstract data structures that interact via procedure calls. Although AO languages frequently inherit the $\mathrm{OO}$ modularity mechanisms of the languages on which they are built [Buck et al. 1994], these mechanisms have largely not been leveraged directly in AO languages. We will show that many (if not all) of the innovations of OO design, including concepts such as the separation of interface from implementation, strong typing of interfaces, subtyping, classes, and inheritance, can be adapted to operate at the level of AO design. We describe an implementation of these mechanisms in Ptolemy II, illustrate the mechanisms with a simple example, and outline their formal structure.

\subsection{Models of Computation}

It is important to realize that the syntactic structure of an actor-oriented language says little about the semantics. The semantics is largely orthogonal to the syntax, and is determined by a model of computation [Lee et al. 2003]. The model of computation might give operational rules for executing a model. These rules determine when actors perform internal computation, update their internal state, and perform external communication. The model of computation also defines the nature of communication between components. In essence, models of computation form design patterns of component interaction, in the same sense that Gamma, et al. describe design patterns in OO languages [Gamma et al. 1994].

Examples of models of computation that have been used in AO languages include the continuous-time semantics of Simulink (from The MathWorks), the dataflow se- 
mantics of LabVIEW (from National Instruments), and the discrete-event semantics of OPNET Modeler (from OPNET Technologies). Although these systems have visual syntaxes and are often viewed more as modeling tools than as programming languages, we consider these software systems to be more akin to editors, interpreters, and compilers for actor-oriented programming languages. Indeed, these systems are increasingly often used as replacements for textual programming languages, particularly in the development of embedded software.

The techniques described in this paper apply broadly to AO design, independent of the model of computation. We have tested them in the Ptolemy II framework with continuous-time, discrete-event, dataflow, and process network semantics, and several more experimental models of computation. They work in all of these because they operate at the level of the abstract syntax, not at the level of the concurrent semantics. Moreover, even without any emphasis on efficiency or scalability, the prototype has proven capable of handling thousands of components efficiently.

\section{RELATED WORK}

\subsection{Software Components}

Prevailing software component architectures such as CORBA, dot Net, and J2EE, are deeply rooted in the procedural semantics of the dominant object-oriented languages $\mathrm{C}++, \mathrm{C} \#$, and Java. In such procedural semantics, concurrency is managed using threads, monitors and semaphores, a notoriously difficult approach [Lee 2006]. As a result, in concurrent systems, it is difficult to treat objects in object-oriented languages as components since they suffer from fragile composition. The interaction between two components can be broken by simply adding more components to the system. Higher-level patterns, such as the CORBA event service, are codified only through object-oriented API, and usage patterns for these APIs are expressed only informally in documentation. The communication mechanism for components becomes an integral part of a component design, making them difficult to reuse.

In actor-oriented abstractions, low-level implementation mechanisms of threads and semaphores do not even rise to consciousness, forming instead the "assemblylevel" mechanisms used to deliver much more sophisticated models of computation. The functionality of components is separated from their communication mechanisms, as is advocated by many researchers [Keutzer et al. 2000; Goessler and Sifakis 2005]. Moreover, actor-oriented abstractions can embrace time and concurrency, and therefore match much better the modeling of embedded systems, which are intrinsically concurrent.

\subsection{Actor-Oriented Design}

Our notion of AO modeling is related to the work of Gul Agha and others. The term actor was introduced in the 1970's by Carl Hewitt of MIT to describe the concept of autonomous reasoning agents [Hewitt 1977]. The term evolved through the work of Agha and others to describe a formalized model of concurrency [Agha 1986; 1990; Agha et al. 1993; Agha et al. 1997]. Agha's actors each have an independent thread of control and communicate via asynchronous message passing. We are further developing the term to embrace a larger family of models of concurrency that are often more constrained than general message passing. Our actors are still

ACM Journal Name, Vol. V, No. N, Month 20YY. 
conceptually concurrent, but unlike Agha's actors, they need not have their own thread of control. Moreover, although communication is still through some form of message passing, it need not be strictly asynchronous. The term "actor" has also been used since the mid 1970s to describe components in dataflow models of computation [Dennis 1974].

A number of more recent efforts adopt an actor-oriented approach. ROOM (Realtime Object-Oriented Modeling [Selic et al. 1994]) from Rational Software (now IBM) extends OO components with ports and concurrent semantics and has influenced the development of "Capsules" in UML-RT and "Composite Structures" in UML-2.* Port-based objects [Stewart et al. 1997], I/O automata [Lynch 1996] and hybrid I/O automata [Lynch et al. 1996], Moses [Esser and Janneck 2001], Metropolis [Goessler and Sangiovanni-Vincentelli 2002], Ptolemy [Buck et al. 1994] and Ptolemy II [Eker et al. 2003] all emphasize actor orientation. Languages for designing actors are a current research topic; for example StreamIT [Thies et al. 2002], which calls actors "filters," and Cal [Eker and Janneck 2003] are languages for designing hardware and software components that interact with dataflow semantics. A number of researchers have also explored heterogeneous mixtures of models of computation [Strehl et al. 2001; Eker et al. 2003; Patel and Shukla 2004; Jantsch and Sander 2005; Herrera and Villar 2006].

\subsection{Prototypes and Classes in Actor-Oriented Languages}

This paper is about extending actor-oriented design techniques with modularity mechanisms like those in $\mathrm{OO}$ languages. A number of interesting experiments in this direction have been performed by others. The GME system from Vanderbilt [Karsai 1995] has been extended to support actor-oriented prototypes [Karsai et al. 2003]. This work is the closest that we have found to what is described in this paper, and we will have more to say about it below.

Some older projects also extend actor-oriented models with modularity methods. CodeSign [Esser 1996], from ETH builds in an OO notion of classes into a design environment based on time Petri nets. Concurrent ML [Reppy 1991], with its synchronous message passing between threads built in a functional style with continuations, can also be viewed as an actor-oriented framework, and has welldeveloped modularity mechanisms. In real-time object-oriented modeling (ROOM) [Selic et al. 1994], ports have protocol roles that are abstract classes defining behavior that the port implements. Each of these mechanisms, however, is tightly bound to a particular concurrent semantics.

The earliest use of object-oriented concepts in actor-oriented design is due to Sutherland [Sutherland 1966]. Some more recent projects build actor-oriented mechanisms on top of languages with object-oriented features, and effectively inherit those features from the host language. Ptolemy Classic, built in $\mathrm{C}++$, is one of the first of these, and the leveraging of object-oriented features is extensively discussed in [Buck et al. 1994]. SystemC, which dates back to Scenic [Liao et al. 1997], is also based on $\mathrm{C}++$, and inherits from the host language object-oriented design capabilities. SML-Sys [Mathaikutty et al. 2004] builds on Standard ML, and hence

*UML had already claimed the term "actors" in use-case diagrams, and hence could not use the term for these concurrent objects.

ACM Journal Name, Vol. V, No. N, Month 20YY. 


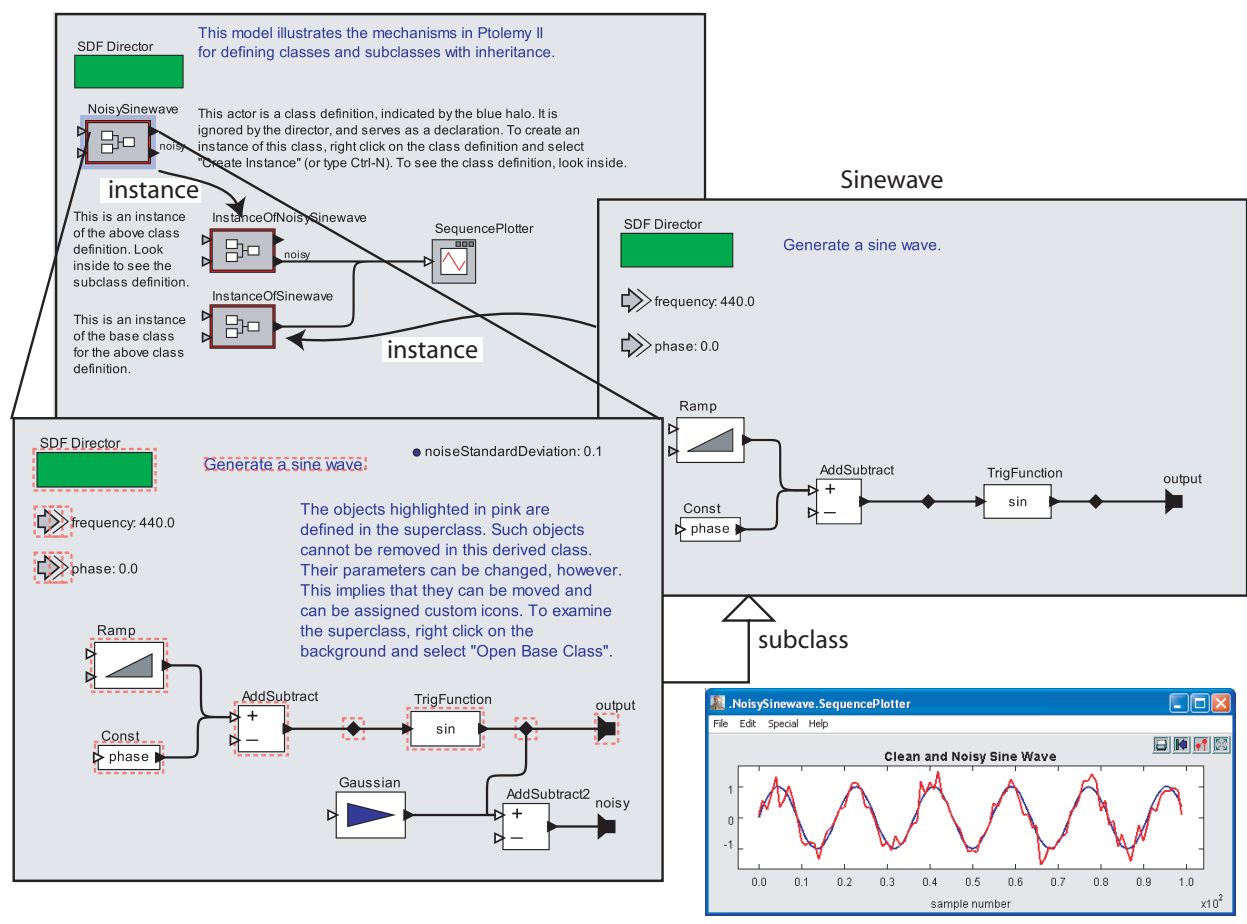

Fig. 2. A simple example of the use of classes in Ptolemy II.

inherits its type system, and most interestingly, its use of higher-order functions. ForSyDe [Sander and Jantsch 2004], Real-Time FRP [Wan et al. 2001], and Lava [Bjesse et al. 1998] similarly build on Haskell. Bluespec [Arvind et al. 2004] derives many of its mechanisms from Haskell, but is now implemented in SystemVerilog. Modelica [Fritzson 2003] provides its own object-oriented mechanisms inspired by functional languages.

This paper is about defining modularity mechanisms for a broad spectrum of actor-oriented semantics. It accomplishes this by defining these mechanisms at the level of the abstract syntax. Our hope is that the next generation of domainspecific frameworks beyond Simulink and LabVIEW will inherit these modularity mechanisms, and that because these mechanisms are independent of the concurrent semantics, designers will become familiar with them and be able to apply them in a wide variety of domain-specific scenarios, as has happened with object-oriented design.

\section{CLASSES IN PTOLEMY ॥}

We begin with a simple example, shown in figure 2 . The model at the top left contains a class definition labeled "NoisySinewave" and an instance of that class labeled "InstanceOfNoisySinewave." The class definition icon has a halo to distinguish it visually from an instance. The NoisySinewave class is defined hierarchically by the model on the lower left. It is a subclass of Sinewave, which is the model 
at the right. NoisySinewave inherits actors, ports, and parameters from Sinewave. The inherited components are outlined with a dashed line, indicating visually that they are inherited components. The NoisySinewave class extends the Sinewave class by adding some additional actors, connections, and ports. These additions do not have the dashed outline.

The model in figure 2, when executed, produces two signal traces, as shown in the plot at the lower right. One is a simple sine wave and the other is a noisy sine wave. The simple sine wave is generated by the InstanceOfSinewave actor, which is an instance of Sinewave, and the noisy sine wave is generated by the InstanceOfNoisySinewave actor, which is an instance of NoisySinewave, a subclass of Sinewave.

In building this mechanism in Ptolemy II, we had to make a number of decisions that amount to language design decisions. The mechanism we have settled on is the one we explain and defend in this paper. However, we remind the reader that mechanisms for object-oriented design took some 40 years to mature. While we can expect faster maturity with actor-oriented design (we have learned from the OO experience), it will not be instantaneous. Our design is one of many possibilities, and while we believe it to be effective and aesthetic, we have no doubt that it can be improved. We explain our mechanism informally first, and then give a precise definition. The precise definition is required to fully grasp the subtleties involved.

First, in Ptolemy II, a model is a set of actors, ports, attributes, and connections. A model can be viewed as a program with a visual syntax. Each of the grey boxes in figure 2 is a model. A special attribute called a director defines the semantics of the model. Each of the models in figure 2 has a director, indicated by the wide (green) rectangle at the upper left in the model. For our purposes here, the director is irrelevant, and can be viewed as any other attribute. The visual annotations in the models are also attributes.

In Ptolemy II, any model can be either a class or an instance. A class serves as a prototype for instances. Our mechanism, therefore, is closely related to prototypebased languages (see chapter 3 of [Craig 2001], for example), but with a twist: the class mechanism operates entirely at the abstract syntax level. As a result, classes in Ptolemy II are purely syntactic objects used for expressing the structure of a model and play no role in the execution of a model. Classes are not visible to the director, which provides the execution engine. As consequence, Ptolemy II does not permit the ports of a class to be externally connected to other ports.

A class may be defined in its own file (in which case we call it a top-level class) or as a component in a model (an inner class). The Sinewave class in figure 2 is a top-level class, while NoisySinewave is not. When a class is defined within a model, its definition is in scope at the same level of the hierarchy where it is defined and at all levels below that. This is the same scoping rule that applies to attributes in the Ptolemy II expression language (see [Brooks et al. 2004]). Thus, for example, the model at the upper left in figure 2 contains both the class definition NoisySinewave and the instance InstanceOfNoisySinewave.

A subclass inherits the structure of its base class. Intuitively, every object (actor, attribute, port or connection) contained by the base class has a corresponding object in the subclass. We refer to this as the derivation invariant. The dashed outlines 
in figure 2 surround such "corresponding objects" provide a visual indication of objects which are defined in a superclass. Ptolemy II disallows removing such objects except through the class definition. However, the subclass can contain new objects and can also change (override) the values of attributes that carry values (we generally refer to attributes that carry values as parameters).

Since a model can contain class definitions, and a model can itself be a class definition, we have inner classes. In Java, C\#, and $\mathrm{C}++$, inner classes are class definitions within class definitions, often with limited scope. In our context, inner classes are class definitions within the model hierarchy, also with limited scope.

Supporting inner classes is a significant departure from the prototype mechanism given in [Karsai et al. 2003], where it is (correctly) pointed out that such inner classes create significant complications. In particular, as we will explain below, they create a specialized form of multiple inheritance. Although this is a significant complication, we believe that it is sufficiently disciplined and expressive to be justified. Moreover, we give a rigorous formalism (based on a generalized ultrametric) that gives inner classes an unambiguous semantics.

Our focus on classes as syntactic constructs led us to several design decisions. Perhaps most importantly, Ptolemy II always represents all objects including instances, classes, and all their contained objects. Although there is significant redundancy between various instances of the same class, we find this not to be terribly wasteful since users are likely to simulate often and all these objects must be elaborated at simulation time. When a model is serialized in a persistant representation, however, we do make use of this redundancy to reduce the amount of data that must be stored. Additionally, notice that all objects in a model must always satisfy the derivation invariant before and after every modification to the design. Ptolemy II provides basic editing operations for constructing models, and in some cases disallows certain operations (such as deleting objects surrounded by a dashed outline) because we did not feel that such operations could be provided for in a way that satisfied the derivation invariant, and was meaningful for a designer.

The rest of this paper develops a formal description of our use of actor-oriented classes in Ptolemy II. We use this description to precisely describe the derivation invariant and the mechanism behind class extension and overriding. We found this formalization to be invaluable in understanding the subtleties that arise when dealing with inner classes defined within another class. We have used the formalism to help develop a comprehensive regression test suite for the software implementation.

\section{FORMAL STRUCTURE}

Figure 3 shows a hierarchy where a top-level model named $\mathbf{T}$ contains four objects, the classes $\mathbf{c A}$ and $\mathbf{c B}$ and their instances $\mathbf{i A}$ and $\mathbf{i B}$. The containment relation is indicated by the solid lines, and parent relation is indicated by the dashed lines. By "parent" we mean either subclassing or instantiation. Thus, $\mathbf{c B}$ is a subclass of $\mathbf{c A}$, while $\mathbf{i B}$ is an instance of $\mathbf{c B}$. We require that objects that share the same container have unique labels, and an individual object within a hierarchy may be referenced by a list of labels following the containment hierarchy. Thus, figure 3 contains five objects with full labels .T, .T.cA, .T.cB, .T.iA, and .T.iB.

ACM Journal Name, Vol. V, No. N, Month 20YY. 


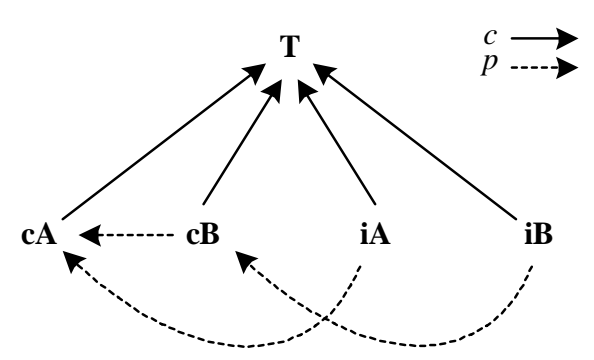

Fig. 3. A model $\mathbf{T}$ containing four objects, the classes $\mathbf{c A}$ and $\mathbf{c B}$ and their instances $\mathbf{i} \mathbf{A}$ and $\mathbf{i B}$. The solid lines denote the container relation, and the dashed lines denote the parent relation.

\subsection{Derivable Objects and Systems}

Let $D$ be the set of derivable objects. These include actors, models (which are actors), attributes and ports. The container relation is a partial function

$$
c: D \rightarrow D
$$

where $c(x)=y$ means that $x$ is contained by $y$. Since this relation is a partial function, a derivable object can have at most one container. When $c(x)=y$ we can also write $(x, y) \in c$, and we say " $x$ is contained by $y . "$

Let $c^{+}$be the transitive closure of the container relation. That is, $(x, y) \in c^{+}$if $(x, y) \in c$ or $(c(x), y) \in c^{+}$. We disallow circular containment, so if $(x, y) \in c^{+}$then $(y, x) \notin c^{+}$. Since $c^{+}$is also irreflexive $\left((x, x) \notin c^{+}\right)$and transitive $\left((x, y) \in c^{+}\right.$ and $\left.(y, z) \in c^{+} \Rightarrow(x, z) \in c^{+}\right)$, then $\left(D, c^{+}\right)$is a strict partially ordered set (strict poset). If $(x, y) \in c^{+}$, we say that " $x$ is deeply contained by $y$."

The parent relation is a partial function

$$
p: D \rightarrow D
$$

where $p(x)=y$ means that either $x$ is a subclass of $y$ or $x$ is an instance of $y$. In either case, we refer to $y$ as the parent and $x$ as the child. Since this is a partial function, a derivable object may have at most one parent. This would seem to rule out multiple inheritance, but as we will see, inner classes provide a disciplined form of multiple inheritance. When $p(x)=y$ we can also write $(x, y) \in p$ and say that " $x$ is a child of $y . "$

Let $p^{+}$be the transitive closure of the parent relation, just as with $c^{+}$. Again, we disallow circular parent relations, so $\left(D, p^{+}\right)$is a strict poset. If $(x, y) \in p^{+}$we say that " $x$ is deeply a child of $y . "$

$\left(D, c^{+}\right)$and $\left(D, p^{+}\right)$are each strict posets. We impose a key additional constraint, which is that $\left(D, c^{+}, p^{+}\right)$is a doubly nested diposet [John Davis 2000], defined as follows.

Definition 1 Doubly Nested Diposet. Given a set $D$ and two strict partial orders $c^{+}$and $p^{+}$, then $\left(D, c^{+}, p^{+}\right)$is a doubly nested diposet if

$$
(x, y) \in c^{+} \Rightarrow(x, y) \notin p^{+} \text {and }(y, x) \notin p^{+}
$$

and

$$
(x, y) \in p^{+} \Rightarrow(x, y) \notin c^{+} \text {and }(y, x) \notin c^{+} .
$$


That is, if $x$ is deeply contained by $y$, then it cannot be (deeply) a child of $y$, nor can $y$ be (deeply) its child. Correspondingly, if $x$ is deeply a child of $y$ then $x$ cannot be deeply contained by $y$ and vice versa. The two partial orders are mutually exclusive.

Let $L$ be the set of all labels. The labeling function is

$$
l: D \rightarrow L
$$

where we require that if $c(x)=c(y)$ and $x \neq y$, then $l(x) \neq l(y)$. The full label of $x$ is the sequence of labels from the top-level container of $x$ (the unique $y$ such that $(x, y) \in c^{+}$and $c(y)$ is undefined) to the label of $x$. We can write this sequence separated by periods. For the example in figure 3 , using the full labels, $(. \mathbf{T . c A}, . \mathbf{T}) \in c$ and $(. \mathbf{T} . \mathbf{i B}, . \mathbf{T . c B}) \in p$. Moreover, $(. \mathbf{T} . \mathbf{i B}, . \mathbf{T . c A}) \in p^{+}$. Note that full labels need not be unique in $D$, so by themselves, they do not provide the identity of an object in $D$.

We collect all our definitions so far into one.

Definition 2 System. A System is a tuple $(D, L, l, c, p)$, where $D$ and $L$ are sets, $l: D \rightarrow L$ is a labeling function, $c: D \rightarrow D$ is a container relation, $p: D \rightarrow D$ is parent relation, and $\left(D, c^{+}, p^{+}\right)$is a doubly nested diposet, where $c^{+}$and $p^{+}$are the transitive closures of $c$ and $p$ respectively.

\subsection{Derived Relation}

The key to our notion of inner classes is the derived relation, which captures a property that follows from the combination of the parent relation and the container relation. An object $x^{\prime}$ is said to be derived from another object $x$ if it is a child of $x$, or if a container somewhere above it is a child such that $x^{\prime}$ exists as a consequence of the derivation invariant.

Definition 3 Derived Relation. Given a system $(D, L, l, c, p)$, let the derived relation $d \subset D \times D$ be the least relation containing $p$ such that $\left(x^{\prime}, x\right) \in d$ implies that either

$$
\left(x^{\prime}, x\right) \in p
$$

( $x^{\prime}$ is a child of $x$ ) or

$$
\left(c\left(x^{\prime}\right), c(x)\right) \in d \text { and } l\left(x^{\prime}\right)=l(x) .
$$

If $\left(x^{\prime}, x\right) \in d$, then we say that " $x^{\prime}$ is derived from $x$." That is, $x^{\prime}$ is derived from $x$ if either $x^{\prime}$ is a child of $x$ or $x^{\prime}$ and $x$ have the same label and the container of $x^{\prime}$ is derived from the container of $x$.

As usual, we let $d^{+}$be the transitive closure relation, and if $\left(x^{\prime}, x\right) \in d^{+}$, we say that " $x$ ' is deeply derived from $x . "$

Consider the example in figure 4. This example has four instances of the class .T.c contained by .T.Y. Each of these instances is derived from .T.c. But more interestingly, the object .T.Y.c1.z is derived from .T.c.z even though there is no parent relation between these.

Definition 4 Implied Relation. Given a system $(D, L, l, c, p)$, we say that $y \in D$ is implied by $z \in D$ if $(y, z) \in d$ and $(y, z) \notin p$. If $y \in D$ is implied by any $z \in D$, then we say that $y$ is implied in the system $S$.

ACM Journal Name, Vol. V, No. N, Month 20YY. 


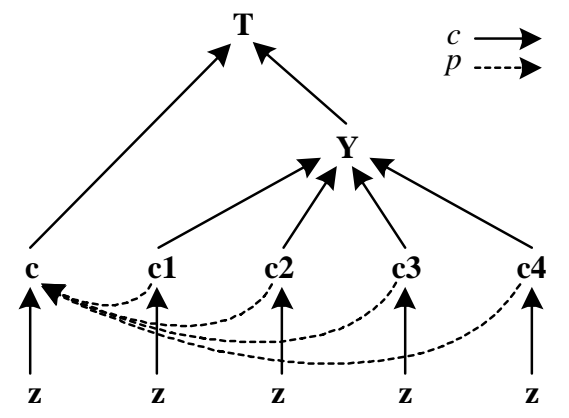

Fig. 4. An example containing one class definition $\mathbf{c}$ and four instances $\mathbf{c 1}$ through $\mathbf{c} 4$. The definition $\mathbf{c}$ is contained by $\mathbf{T}$, whereas the instances are contained by $\mathbf{Y}$, which is in turn contained by $\mathbf{T}$.

Thus, in figure 4, .T.Y.c1.z is implied by .T.c.z. If $y$ is implied by $z$, then it is not necessary to represent $y$ explicitly in a persistent representation of the model which contains $z$ (unless it overrides $z$ in some way, as we will discuss). This is because the derivation invariant and the presence of $z$ implies the existence of $y$, and hence, any tool that reads the persistent representation will construct $y$ simply as a consequence of constructing $z$. The ability to omit components from a persistent representation can be very helpful. Even for some relatively simple and practical Ptolemy II models, we have found that the XML representation of the model could shrink by a factor of 10 or more, if we omit implied objects. However, it is imperative that we accurately exclude only those components that are implied. This turns out to be particularly challenging when persistent representations need to be generated for only a portion of a model (for example, in copy-and-paste functionality, where a portion of a model is copied and then pasted elsewhere into another model). To get this right, we need to formalize the derivation invariant.

\subsection{Derivation Invariant}

We will require that well-formed models satisfy a key structural property that we call the "derivation invariant." This property is our key constraint on the structure of models; it has a number of attractive consequences. Informally, the derivation invariant states that if $x^{\prime}$ is derived from $x$, then $x^{\prime}$ has at least the structure of $x$. We give a formal definition.

Definition 5 Derivation Invariant. Given a system $(D, L, l, c, p)$, let $d$ be the derived relation as given in definition 3. Then the system satisfies the derivation invariant if $\left(x^{\prime}, x\right) \in d$ implies that the following property holds:

For all $z$ where $c(z)=x$, there exists a $z^{\prime}$ where $c\left(z^{\prime}\right)=x^{\prime}$ and $l(z)=$ $l\left(z^{\prime}\right)$ and either

(1) $p(z)$ and $p\left(z^{\prime}\right)$ are undefined, or

(2) $\left(p\left(z^{\prime}\right), p(z)\right) \in d$ and $c(p(z))=x$ and $c\left(p\left(z^{\prime}\right)\right)=x^{\prime}$, or

(3) $p(z)=p\left(z^{\prime}\right)$ and both $(p(z), x) \notin c^{+}$and $\left(p\left(z^{\prime}\right), x^{\prime}\right) \notin c^{+}$.

The primary implication is that if $x^{\prime}$ is derived from $x$, then for every $z$ contained by $x$ there is a corresponding $z^{\prime}$ contained by $x^{\prime}$ with the same label. Clearly by 
the definition of $d,\left(z^{\prime}, z\right) \in d$. This means that $x^{\prime}$ contains (deeply) an object corresponding to each object contained (deeply) by $x$.

The three alternative cases in definition 5 give the information necessary to recreate the parent of $z^{\prime}$. The first alternative states that if $z$ has no parent, then $z^{\prime}$ also has no parent. The second alternative states that if $z$ has a parent and its parent is contained by $x$, then the parent of $z^{\prime}$ is the object in $x$ implied by the parent of $z$. The third alternative states that if $z$ has a parent, but that parent is not contained in $x$, then $z^{\prime}$ has the same parent as $z$, and that parent is also not contained by $x^{\prime}$. In Ptolemy II, for example, that parent might be a top-level class.

In practice, derived objects may have more similarity than is implied by simply having the same "label." Formally, since we have not given any semantics to a label, this constraint can emcompass any measure of similarity that we wish. In Ptolemy II, for example, derived components are required to be represented by instances of the same Java class. That is, if $x^{\prime}$ is derived from $x$, then $x^{\prime}$ must be an instance of the same Java class (or a derived Java class) that $x$ is an instance of. This binds the $\mathrm{AO}$ design structure to the $\mathrm{OO}$ design structure in very useful ways.

\subsection{Derivation Depth}

The derivation invariant imparts particularly useful structure to a model. For example, in a system that satisfies the derivation invariant, for any $\left(z^{\prime}, z\right) \in d$, exactly one of the two possible conditions in definition 3 is true, as indicated by the following lemma.

Lemma 1. Given a system $(D, L, l, c, p)$ that satisfies the derivation invariant, for any $\left(z^{\prime}, z\right) \in d$, exactly one of the two following conditions is true:

(1) $\left(z^{\prime}, z\right) \in p$ or

(2) $\left(c\left(z^{\prime}\right), c(z)\right) \in d$ and $l\left(z^{\prime}\right)=l(z)$.

Proof. By definition 3, the two conditions cannot both be false. Therefore, we need only show that they cannot both be true. To do this, show that if the first condition is true, i.e. that $p\left(z^{\prime}\right)=z$, then the second is false. In this case, the derivation invariant implies that one of the two following conditions must be true:

(a) $\left(p\left(z^{\prime}\right), p(z)\right) \in d$ and $(p(z), c(z)) \in c$ and $\left(p\left(z^{\prime}\right), c\left(z^{\prime}\right)\right) \in c$, or

(b) $p(z)=p\left(z^{\prime}\right)$ and both $(p(z), c(z)) \notin c^{+}$and $\left(p\left(z^{\prime}\right), c\left(z^{\prime}\right)\right) \notin c^{+}$.

Condition (b) clearly cannot hold because $p\left(z^{\prime}\right)=z$ and by the definition of $p$ it cannot be true that $p(z)=z$. We can rewrite condition (a), using $p\left(z^{\prime}\right)=z$, as

$$
(z, p(z)) \in d \text { and } c(p(z))=c(z)=c\left(z^{\prime}\right) .
$$

If this condition holds, then it cannot be true that $\left(c\left(z^{\prime}\right), c(z)\right) \in d$ because $c\left(z^{\prime}\right)=$ $c(z)$. Thus, it cannot be true that both condition (1) and (2) hold.

The following corollary is easily shown by induction.

COROLlary 1. Given a system $(D, L, l, c, p)$ that satisfies the derivation invariant, for any $\left(z^{\prime}, z\right) \in d$, there is a least $n \in \mathbb{N}$, where $\mathbb{N}=\{0,1,2, \ldots\}$ is the set of natural numbers, such that

$$
\left(c^{n}\left(z^{\prime}\right), c^{n}(z)\right) \in p,
$$

ACM Journal Name, Vol. V, No. N, Month 20YY. 


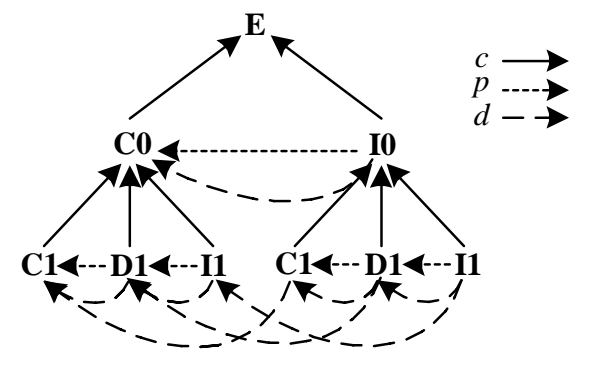

Fig. 5. A model $\mathbf{E}$ with inner classes.

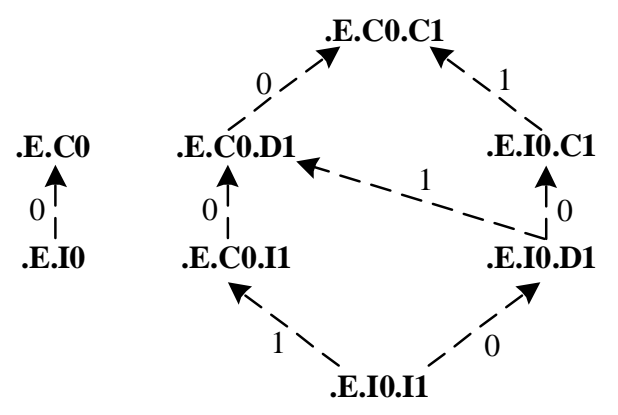

Fig. 6. Derived relation among .E.C0.C1, .E.C0.I1, .E.I0.C1, and .E.I0.I1.

where we assume $c^{0}(z)=z$, and for all $i \in \mathbb{N}$ where $0<i \leq n, l\left(c^{n-1}\left(z^{\prime}\right)\right)=$ $l\left(c^{n-1}(z)\right)$.

Definition 6 Derivation Depth. The derivation depth is a partial function

$$
h: D \times D \rightarrow \mathbb{N}
$$

defined for every $\left(x^{\prime}, x\right) \in d$ to be equal to the least $n$ of corollary 1.

That is, the derivation depth tells us, for a pair $x^{\prime}$ derived from $x$, how far above in the containment hierarchy is the parent relation that leads to the derived relation.

\subsection{Subclassing, Inheritance, and Overriding}

Any object that is the parent of another is, analogous to OO design, a class definition. The child is either an instance or a subclass, depending on whether it too is a parent. The derivation invariant provides a form of inheritance. In particular, if $x^{\prime}$ is derived from $x$, then $x^{\prime}$ inherits contained objects matching those in $x$. We can use the derived relation to cleanly define overriding, which allows for AO subclassing. In particular, if $x^{\prime}$ is derived from $x$, the derivation invariant does not prevent $x$ from containing additional objects that have no corresponding object in $x$. The NoisySinewave subclass in figure 2 contains just such additional objects. When a subclass has such additional objects, it has overridden the structure it inherited.

Figure 5 shows a model with inner classes. The top level object is .E. It contains a class (also an object) .E.C0 and an instance .E.I0 of that class. The class contains 
another class .E.C0.C1, a subclass .E.C0.D1, and an instance .E.C0.I1. The instance .E.I0 contains three implied objects, .E.I0.C1, .E.I0.D1, and .E.I0.I1. The container relation is shown with solid lines, the parent relation with dotted lines, and the derived relation with dashed lines. The derived relation alone is extracted and redrawn for insight in figure 6 . The numbers annotating the arrows are the derivation depth of definition 6 .

For example, in figure 6 ,

$$
h(. \mathbf{E} . \mathbf{C 0 . C 1} . \mathbf{E . C 0 . D 1 )}=0
$$

because (.E.C0.C1,.E.C0.D1) $\in p$, or .E.C0.C1 is a parent of .E.C0.D1. Correspondingly,

$$
h(. \mathbf{E . C 0 . C 1 , . E . I 0 . C 1 )}=1
$$

because the container of .E.C0.C1 is a parent of the container of .E.I0.C1 and the two have the same label. Moreover, $h($.E.C0.C1,.E.I0.I1) is undefined because although (.E.C0.C1,.E.I0.I1) $\in d^{+}$, it is not in $d$. The derivation depth will help us to resolve a form of multiple inheritance, explored in the next subsection.

\subsection{Valuation and Inherited Values}

We now explore another form of inheritance that proves extremely useful. In particular, certain objects in a model have values. For example, parameters of an actor have values. Let the valuation be a partial function

$$
v: D \rightarrow V
$$

where $V$ is a set of values. We assume that values are also inherited and can be overridden. A key issue is to determine whether $\left(x^{\prime}, x\right) \in d^{+}$implies that $v\left(x^{\prime}\right)=$ $v(x)$. This question relates to inheritance, but is somewhat more complicated than the structural inheritance described above. In particular, a subclass may override the value of an object, and that override may shadow further derived objects. It is precisely this complication that lead the authors of [Karsai et al. 2003] to disallow inner classes. We have taken a more aggressive stand, which is to allow subclasses and to give a clean semantics to overriding. This choice has proven useful in a number of practical Ptolemy II designs. It certainly enriches the model, and makes it much more modular, since classes can locally contain class definitions.

Multiple inheritance occurs because we can have $x \neq y$ and $\left(x^{\prime}, x\right) \in d^{+}$and $\left(x^{\prime}, y\right) \in d^{+}$. Should $x^{\prime}$ inherit its value from $x$ or $y$ ? Intuitively, we resolve this dilemma by asserting that it should inherit from whichever is closest. The challenge is to define "closest." We will do this by constructing a generalized ultrametric space with the remarkable property that if $x^{\prime}$ is (deeply) derived from any two objects, then one of those is always strictly closer than the other. Multiple inheritance can always be unambiguously resolved.

A metric gives a distance between two objects as a non-negative real number. A metric satisfies the triangle inequality (the distance between $a$ and $c$ is less than or equal to the sum of the distance between $a$ and $b$ and the distance between $b$ and $c$ ). An ultrametric satisfies a stronger condition (the distance between $a$ and $c$ is less than or equal to the greater of the distances between $a$ and $b$ and between $b$

ACM Journal Name, Vol. V, No. N, Month 20YY. 
and c). A generalized ultrametric [Priess-Crampe and Ribenboim 1996] measures distance not as a real number, but rather as an element in a totally ordered set.

Our totally ordered set will be $S$ be given by

$$
S=\mathbb{N}^{*} \cup\{\top\}
$$

where $\mathbb{N}^{*}$ is the set of all finite sequences of natural numbers and $T$ is a special element that we will take to be greater than all such sequences. We write [ ] $\in S$ to represent the empty sequence and $[0,2]$ to represent a sequence with two natural numbers, 0 followed by 2 . We now define an ordering relation on $S$.

Definition 7 Distance Order. Define a distance order $\prec$ on $S$ as follows. First,

$$
s_{1} \prec \top \quad \forall s_{1} \neq \top .
$$

Next, for any $s_{1}=\left[a_{0}, \ldots, a_{m}\right]$ and $s_{2}=\left[b_{0}, \ldots, b_{n}\right], s_{1} \prec s_{2}$ if $m<n$. That is, a shorter sequence is less than a longer sequence. If the sequences have the same length, $m=n$, then there is a maximal $k \leq m$ such that $b_{i}=a_{i}$ for all $i, 0 \leq i<k$, and we define $s_{1} \prec s_{2}$ if $a_{k}<b_{k}$. That is, we compare the earliest elements of $s_{1}$ and $s_{2}$ that differ, and the sequence with the smaller one is less than the sequence with the larger one. In all other circumstances, unless $s_{2}=s_{1}$, we define $s_{2} \prec s_{1}$.

For example,

$$
[] \prec[2] \prec[0,1] \prec[2,1] \prec \top .
$$

The following lemma is trivial.

Lemma 2. The relation $\prec$ is a total order on $S$.

We further define a max-plus algebra [Baccelli et al. 1992] on $S$ as follows. Let the operator $\otimes: S \times S \rightarrow S$ be the pointwise sum of sequences. For example,

$$
[1,2] \otimes[3,4]=[4,6] .
$$

If the sequences do not have equal lengths, then we implicitly extend the shorter one with zeros, so that for example

$$
[1] \otimes[3,4]=[4,4] .
$$

As a consequence, the empty sequence is the additive identity,

$$
[] \otimes s=s, \quad \forall s \in S .
$$

As a special case,

$$
s \otimes \top=\top \otimes s=\top, \quad \forall s \in S .
$$

Note that $\otimes$ is commutative and associative. Moreover, for all $s_{1}, s_{2} \in S$,

$$
s_{1} \preceq s_{1} \otimes s_{2} \text { and } s_{2} \preceq s_{1} \otimes s_{2} .
$$

We further define the operator $\oplus: S \times S \rightarrow S$ to yield the minimum in the distance order. That is

$$
\begin{aligned}
s_{1} \oplus s_{2}= \begin{cases}s_{1} & \text { if } s_{1} \prec s_{2} \\
s_{2} & \text { otherwise }\end{cases} \\
\text { ACM Journal Name, Vol. V, No. N, Month 20YY. }
\end{aligned}
$$




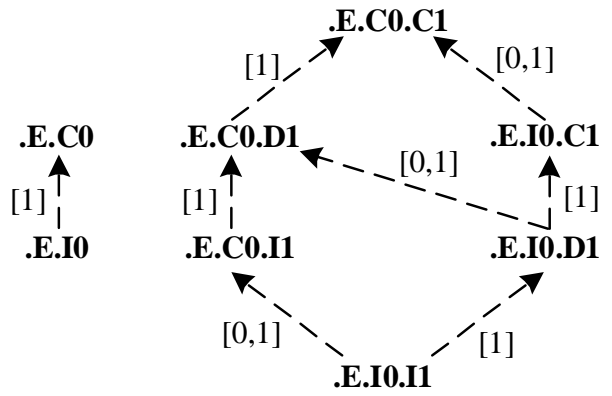

Fig. 7. Derived relation as in figure 6 but with the edges labeled with the distance function $s$.

Note that $\oplus$ is commutative, associative, and idempotent. Moreover, $\otimes$ is distributive over $\oplus$,

$$
s_{1} \otimes\left(s_{2} \oplus s_{3}\right)=\left(s_{1} \otimes s_{2}\right) \oplus\left(s_{1} \otimes s_{3}\right) .
$$

To define the distance function, we will use the graph of the derived relation, as in figure 6 , but we will relabel the arcs with weights in $S$ rather than in $\mathbb{N}$. For the example in figure 6 , we have done such relabeling in figure 7 , where we define the weights to be

$$
w(x, y)=[\underbrace{0, \cdots, 0}_{n}, 1]
$$

for any if $(x, y) \in d$, where $n$ is the derivation depth of definition 6 .

We can now define a distance function $s: D \times D \rightarrow S$ that will prove to be a generalized ultrametric. First, we declare that if $(x, y) \notin d^{+}$and $(y, x) \notin d^{+}$, then

$$
s(x, y)=s(y, x)=\top .
$$

We interpret this to mean that the distance between two objects that are unrelated by derivation is "infinite." Next we define

$$
s(x, x)=[] .
$$

That is, the distance of any object to itself is "zero" (the additive identity). For any $(x, y) \in d$, we define $s(x, y)=w(x, y)$.

It only remains to define the distance function for $(x, y) \in d^{+}$but $(x, y) \notin d$. For each such $(x, y)$, there is at least one directed path from $x$ to $y$ in the graph of the derived relation, as in figure 7. Let the set of all such paths from $x$ to $y$ be denoted $\operatorname{paths}(x, y)$. A path $P \in \operatorname{paths}(x, y)$ is the set of pairs of objects $(u, w) \in d$ along the path. For any $(x, y) \in d^{+}$we now define the distance

$$
s(x, y)=s(y, x)=\bigoplus_{P \in \mathbf{p a t h s}(x, y)}\left(\bigotimes_{(u, w) \in P} w(u, w)\right) .
$$

Consider the example in 7. From this picture, we can determine the distance $s$ ACM Journal Name, Vol. V, No. N, Month 20YY. 
between any two objects. For example,

$$
\begin{aligned}
s(. \mathbf{E . I 0 . I 1}, \text { E.C0.D1 }) & =([0,1] \otimes[1]) \oplus([1] \otimes[0,1]) \\
& =[1,1] \oplus[1,1] \\
& =[1,1] .
\end{aligned}
$$

Here are a few more examples:

$$
\begin{aligned}
& s(. \mathbf{E . I 0 . I 1}, \mathbf{E . C 0 . C 1})=[2,1] \\
& s(. \mathbf{E . I 0 . I 1} \text {.E.C0.I1) }=[0,1] \\
& s(. \text { E.IO.I1,.E.IO.C1) }=[2]
\end{aligned}
$$

For $(x, y) \in d^{+}, s(x, y)$ always has its last value greater than zero (note that the special case $\left.(x, x) \notin d^{+}\right)$.

We next prove that $s$ is a generalized ultrametric, and thus a reasonable measure of distance. To be a generalized ultrametric [Priess-Crampe and Ribenboim 1996], $s$ must satisfy three properties. For all $x, y \in D$,

(1) $s(x, y)=[] \Leftrightarrow x=y$

(2) $s(x, y)=s(y, x)$

(3) $s(x, y) \preceq \max (s(x, z), s(z, y)), \quad \forall y \in D$.

THEOREM 1. Given a system $(D, L, l, c, p)$ that satisfies the derivation invariant, the function s given by (3) is a generalized ultrametric.

Proof. The first two properties follow trivially from the definition. To show the third, we consider several cases. First, if $x=y$, then $s(x, y)=[]$, the bottom element in the order, so the result follows. If there is no path from $x$ to $y$ or $y$ to $x$, then $s(x, y)=\top$, but it must also be true that for all $z \in D$ there is no path from $x$ to $z$ or no path from $z$ to $y$, so $\max (s(x, z), s(z, y))=\top$, and again the result follows. For the case where there is a path from $x$ to $y$, we only need to consider those $z \in D$ where there is a path from $x$ to $z$ and from $z$ to $y$ (otherwise, the right hand side has value $T$, and the result follows trivially). In this case, from the definition of $s$, we see that

$$
s(x, y) \preceq s(x, z) \otimes s(z, y) .
$$

From this and (1), the result follows.

The ultrametric helps us resolve the multiple inheritance question. Specifically, if $x \neq y$ and $\left(x^{\prime}, x\right) \in d^{+}$and $\left(x^{\prime}, y\right) \in d^{+}$, should $x^{\prime}$ inherit its value from $x$ or $y$ ? Our answer is that it should inherit from $x$ if $s\left(x^{\prime}, x\right) \prec s\left(x^{\prime}, y\right)$. But what if $s\left(x^{\prime}, x\right)=s\left(x^{\prime}, y\right)$ ? We will now develop a theorem that shows, remarkably, that this cannot occur.

THEOREM 2. Given a system $(D, L, l, c, p)$ that satisfies the derivation invariant, if $x \neq y$ and $\left(x^{\prime}, x\right) \in d^{+}$and $\left(x^{\prime}, y\right) \in d^{+}$, then $s\left(x^{\prime}, x\right) \neq s\left(x^{\prime}, y\right)$.

ProOF. If either $(x, y) \in d^{+}$or $(y, x) \in d^{+}$, then the theorem follows easily. Assume without loss of generality the first of these. Then

$$
s\left(x^{\prime}, y\right)=s\left(x^{\prime}, x\right) \otimes s(x, y) \succ s\left(x^{\prime}, x\right)
$$

from (1). Hence the theorem holds in this case. 


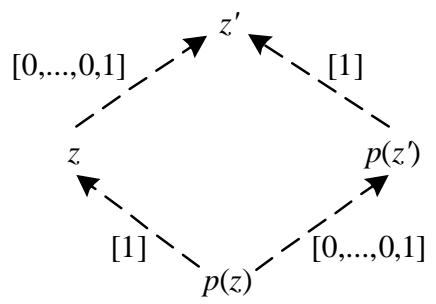

(2)

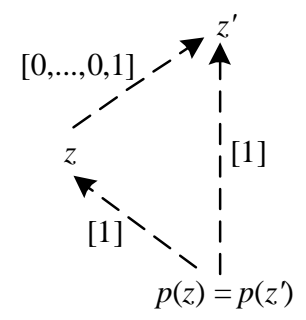

(3)

Fig. 8. Derived relations when alternatives (2) or (3) of the derivation invariant hold.

When $(x, y) \notin d^{+}$and $(y, x) \notin d^{+}$, the proof is considerably more difficult. Our proof will use properties of the graph of the derived relation, like that in figure 7 . First note that to construct this graph, we can define the nodes of the graph to be all the objects in a model. Whenever there is a pair of objects with a parent-child relationship, $\left(x^{\prime}, x\right) \in p$, then there is a directed edge from $x$ to $x^{\prime}$ with weight [1]. If these were the only edges, then the graph would be a forest (a set of trees), since no object can have more than one parent. But these are not the only edges in the graph. There are also edges due to the implied relation. I.e., if $\left(z^{\prime}, z\right) \in d$ but $\left(z^{\prime}, z\right) \notin p$, then there is an edge from $z$ to $z^{\prime}$ with weight of the form $[0, \cdots 0,1]$, with at least one leading 0 . These edges are induced by the derivation invariant. Recall from definition 5 that if $\left(x^{\prime}, x\right) \in d$, then for every $z$ contained by $x$ there is a $z^{\prime}$ contained by $x^{\prime}$ that is implied by $z$. Hence there is an edge from $z$ to $z^{\prime}$. Recall further that $z$ and $z^{\prime}$ must satisfy one of the following three conditions:

(1) $p(z)$ and $p\left(z^{\prime}\right)$ are undefined, or

(2) $\left(p\left(z^{\prime}\right), p(z)\right) \in d$ and $c(p(z))=x$ and $c\left(p\left(z^{\prime}\right)\right)=x^{\prime}$, or

(3) $p(z)=p\left(z^{\prime}\right)$ and both $(p(z), x) \notin c^{+}$and $\left(p\left(z^{\prime}\right), x^{\prime}\right) \notin c^{+}$.

If all such $z$ satisfy the first condition, then the derived graph is a forest. When the derived graph is a forest, the theorem follows easily because $x \neq y$ and $\left(x^{\prime}, x\right) \in d^{+}$ and $\left(x^{\prime}, y\right) \in d^{+}$implies that either $(x, y) \in d^{+}$or $(y, x) \in d^{+}$. Assume without loss of generality the first of these. Then

$$
s\left(x^{\prime}, y\right)=s\left(x^{\prime}, x\right) \otimes s(x, y) \succ s\left(x^{\prime}, x\right)
$$

from (1). Hence the theorem is established for the situation where only option (1) above holds.

When options (2) and (3) hold, the situation is more complicated because the derived graph is no longer a forest. These options each induce the structures shown in figure 8 , where the edges with weights labeled $[0, \cdots, 0,1]$ each have at least one leading 0 . The key to our proof is that these structures, and these structures alone, make the graph no longer a forest. In particular, these structures provide the only two mechanisms by which a node in the graph can have two incoming edges.

To prove the theorem when structures like those in figure 8 are found, consider $\left(x^{\prime}, x\right) \in d^{+}$and $\left(x^{\prime}, y\right) \in d^{+}$where $x \neq y$ and $(x, y) \notin d^{+}$and $(y, x) \notin d^{+}$. This means that if we traverse the graph backwards (starting from $x^{\prime}$ and moving 
upstream along directed edges), we must encounter at least one of the two structures in figure 8, or the path could not fork to two distinct $x$ and $y$. Suppose a fork occurs at a node $z^{\prime}$ along the path from $x^{\prime}$ to either $x$ or $y$. If $\left(z^{\prime}, x\right) \in d^{+}$, then we always choose the right-hand path in figure 8 to get to $x$. Otherwise, we choose the left path. If at each such fork we make this choice we will be assured that the path weight is minimal, and hence the path weight will be equal to $s\left(x^{\prime}, x\right)$. This amounts to a greedy algorithm, where at each fork we choose the minimal weight branch if that branch can still lead back to $x$.

In order for there to be a path from $x^{\prime}$ back to two distinct $x$ and $y$, where $(x, y) \notin d^{+}$and $(y, x) \notin d^{+}$, there must be a fork at some $z^{\prime}$ where the greedy choice is not possible for one of $x$ or $y$. Assume without loss of generality that it is not possible for $y$. That is, $\left(p\left(z^{\prime}\right), y\right) \notin d^{+}$and $p\left(z^{\prime}\right) \neq y$. In this case, it must also be true that $(p(z), y) \notin d^{+}$and $p(z) \neq y$. Hence, with either structure in figure 8 , we need either that $y=z$ or there must be another fork at $z$. Moreover, at that other fork it must again be true that the greedy path is not available. Hence, all remaining edges on the path to $y$ have weights of the form $[0, \cdots, 0,1]$, with a zero in the first position. If $x \neq y$, then the path to $x$ must have been able to take the greedy branch at at least one of these forks. Thus, the path weight to $x$ (and hence the distance $s\left(x^{\prime}, x\right)$, must differ by at least 1 in the first position from the path weight to $y$ (and hence the distance $s\left(x^{\prime}, y\right)$. Hence, the theorem is established.

To see how to apply this theorem, consider the example in figure 5. Suppose that that valuations $V$ are integers, and that $v(. \mathbf{E . C 0 . C 1})=10$. If none of the other objects in figure 6 overrides the value, then all will have value 10. Suppose, however, that $v(. \mathbf{E . C 0 . I 1})=9$, an override. Then $v($ E.I0.I1 $)=9$, inheriting the override. Suppose, however, that $v($.E.I0.D1 $)=8$, another override. This latter override is "closer", yielding $v($.E.I0.I1 $)=8$.

\subsection{Implementation of the Override Mechanism}

An efficient implementation of this mechanism is challenging. It requires tracking which values are overrides, and also requires avoiding traversing the entire graph of derived relations each time a value is needed. We have constructed in Ptolemy II an implementation that has linear complexity. It relies on the fact that values are assigned to objects sequentially when a model is constructed, and that as these values are assigned, the valuation function $v$ evolves towards its final form.

DEFINITION 8 OverRIDE FunCtion. Let the override function be a partial function

$$
r: D \rightarrow S
$$

where for each $x^{\prime} \in D, r(d)$ specifies the priority of the path that has determined the current value of $x^{\prime}$, if there is such a value and path and $\top$ otherwise. Specifically, $r\left(x^{\prime}\right)=$ [] if its value is set directly (an override), and $r\left(x^{\prime}\right)=s\left(x^{\prime}, x\right)$ if its value is set due to propagation of a value from $x$.

The mechanism is easiest to understand if one visualizes the construction of a model, which involves sequentially assigning values to objects. As a model is constructed, the definition of the function $r$ changes as the function $v$ changes, because it changes when values change. In particular, initially, $r$ is undefined for 
all $x \in D$. When a value is set for some $x^{\prime}$, the value may propagate to derived objects. We use $r$ to track whether a value was set directly or via propagation, and if it was via propagation, then what the override strength of the value is.

We can now determine whether a change to the value of $x^{\prime}$ should propagate to $x$. If $\left(x^{\prime}, x\right) \notin d^{+}$, then it should not. If $\left(x^{\prime}, x\right) \in d^{+}$but $r\left(x^{\prime}\right)$ is undefined, then it should, so that $v\left(x^{\prime}\right)=v(x)$. If $\left(x^{\prime}, x\right) \in d^{+}$but $r(x)=[]$, then it should not, because $x$ has been previously set directly. If $\left(x^{\prime}, x\right) \in d^{+}$but $s\left(x^{\prime}, x\right) \prec r(x)$, then it should, because $x$ has inherited a (propagated) value, but the propagation occurred higher up in the containment hierarchy or more remote over the parentchild relation than the current one. If propagation occurs, then together with the change in in $v(x)$, we must redefine the function $r$ so that $r(x)=s\left(x^{\prime}, x\right)$.

\subsection{Dynamic Structure}

We assume that the structure of a model and the valuation of objects with values can change during execution of the model. In fact, the above mechanism for propagating valuations supports dynamically changing valuations. But in addition, Ptolemy II supports dynamically changing model structure. That is, new instances can be created, new subclasses can be defined, and new classes can be defined. In fact, as we construct a model, at each step of the construction we will have a coherent model, so model construction and model mutation are indistinguishable. Each change to the model will represent a change to the key relations $c, p$, $v$, and $r$. We assume that such changes are atomic and sequential, and that after every change, the derivation invariant remains true. It is, of course, a challenge in the design of the Ptolemy II software to ensure that this is true, particularly since the software system is intrinsically highly concurrent. The mechanism that ensures sequential and atomic model mutations is documented in [Brooks et al. 2004].

\subsection{Persistent Representation}

Suppose we are given a system that satisfies the derivation invariant. Suppose further that we wish to construct a persistent representation of a particular component $y$ and all of its contents within this system (for example, to perform a copy-andpaste operation within a model editor). A key question arises: what portion of the system should be represented? The following definition helps to answer this question.

Definition 9 Downset on the Container Relation. Given a system $(D, L, l, c, p)$, let the downset function on the container relation be a function

$$
\downarrow_{c}: D \rightarrow P(D),
$$

where $P(D)$ is the powerset of $D$, such that

$$
x \in \downarrow_{c}(y) \Longleftrightarrow(x, y) \in c^{+} \text {or } x=y .
$$

The motivation for this definition is that a persistent representation of $y$ does not need to include anything that is not in $\downarrow_{c}(y)$. That is, $\downarrow_{c}(y)$ is a complete description of $y$ and everything it contains.

However, $\downarrow_{c}(y)$ may be a bigger description than necessary. In particular, consider again the example in figure 4 . Each of the instances .T.Y.ci.z, for 
$i \in\{1, \cdots, 4\}$, is implied by .T.c.z. Hence, although there are 12 objects in figure 4 , four of them can be omitted in any persistent description of the system because they are implied. In practice, with Ptolemy II models, we have seen dramatic reductions of file sizes for representing models.

However, suppose we wish to construct a persistent representation of $y=\mathbf{. T . Y}$ and everything it contains. The set $D_{y}=\downarrow_{c}(y)$ has 21 elements, none of which is implied by any other element in the set. If we include .T.c in the description of $y$, then we do not need to include all 21 elements. It would be sufficient to include only 11 . We now give a systematic method to construct such a reduced set, thus leveraging the derivation invariant to come up with dramatically more compact descriptions of models.

Definition 10 Upset on the (Deep) Derived Relation. Given a system $(D, L, l, c, p)$, let the upset function on the (deep) derived relation be a function

$$
\uparrow^{+}: D \rightarrow P(D)
$$

such that

$$
x \in \uparrow^{d^{+}}(y) \Longleftrightarrow(y, x) \in d^{+} \text {or } x=y .
$$

For example, in figure 4,

$$
\uparrow^{d^{+}}(. \mathbf{T} . \mathbf{Y} . \mathbf{c 1})=\{. \mathbf{T} . \mathbf{c}\} .
$$

Both the downset and upset functions can be lifted and applied to sets. For example, we define

$$
\downarrow_{c}: P(D) \rightarrow P(D)
$$

so that for all $D^{\prime} \subset D$,

$$
\downarrow_{c}\left(D^{\prime}\right)=\cup_{y \in D^{\prime}} \downarrow_{c}(y)
$$

and similarly for the upset.

As a shorthand, define the upset downset function by

$$
\uparrow_{c}^{d^{+}}: D \rightarrow P(D)
$$

such that

$$
\uparrow_{c}^{d^{+}}(y)=\downarrow_{c} \uparrow^{d^{+}} \downarrow_{c}(y) .
$$

The significance of the upset downset is that it contains all the information that is needed to reconstruct the model (deeply) contained by $y$, preserving implied relations. The upset downset function can similarly be lifted to apply to sets.

We will be interested in minimal descriptions of subsystems.

Definition 11 Subsystem. Given a system $S=(D, L, l, c, p)$ and a subset $U \subset$ $D$, let $l_{U}, c_{U}$, and $p_{U}$ be the relations $l, c$, and $p$ restricted to $U$. Then the system $S_{U}=\left(U, L, l_{U}, c_{U}, p_{U}\right)$ is a subsystem of $S$.

The following property now follows immediately from the definitions.

Property 1. Given a system $S=(D, L, l, c, p)$, for any subset $D^{\prime} \subset D$, let $U=\uparrow_{c}^{d^{+}}\left(D^{\prime}\right)$ and $S_{U}$ be the corresponding subsystem. Then if $x^{\prime} \in D^{\prime}$ is implied in $S$, then $x^{\prime}$ is implied in the subsystem $S_{U}$.

ACM Journal Name, Vol. V, No. N, Month 20YY. 
A consequence of this property is that if we wish to describe a system containing only a subset $D^{\prime} \subset D$ of objects, we can instead describe the subsystem $S_{U}$ for $U=\uparrow_{c}^{d^{+}}\left(D^{\prime}\right)$, and in this subsystem, we can omit any objects that are implied in the original system. They will be implied in the subsystem.

Another key property the subsystem $S_{U}$ for $U=\uparrow_{c}^{d^{+}}\left(D^{\prime}\right)$ is that it is internally self-consistent in that it satisfies the derivation invariant if the original system satisfies the derivation invariant.

TheOREM 3. Given a system $S=(D, L, l, c, p)$ that satisfies the derivation invariant, let $U=\uparrow_{c}^{d^{+}}\left(D^{\prime}\right)$ for some subset $D^{\prime} \subset D$ and let $S_{U}$ be the corresponding subsystem. If $S$ satisfies the derivation invariant, then so does $S_{U}$.

Proof. First we show that if $\left(x^{\prime}, x\right) \in d$ and either $x^{\prime} \in D^{\prime}$ or $x \in D^{\prime}$, then $\left(x^{\prime}, x\right) \in d_{U}$. To show this, note that

$$
x^{\prime} \in D^{\prime} \Rightarrow x \in \uparrow_{c}^{d^{+}}\left(D^{\prime}\right) \text { and } x \in D^{\prime} \Rightarrow x^{\prime} \in \uparrow_{c}^{d^{+}}\left(D^{\prime}\right) .
$$

Thus,

$$
x^{\prime} \in D^{\prime} \text { or } x \in D^{\prime} \Rightarrow x \in \uparrow_{c}^{d^{+}}\left(D^{\prime}\right) \text { and } x^{\prime} \in \uparrow_{c}^{d^{+}}\left(D^{\prime}\right) .
$$

Second, note that for any subset $D^{\prime} \subset D$ and $U=\uparrow_{c}^{d^{+}}\left(D^{\prime}\right)$, if $\left(x^{\prime}, x\right) \in c^{+}$and $x^{\prime} \in U$ and $x \in U$, then $\left(x^{\prime}, x\right) \in c_{U}^{+}$. The same is true of the deep parent relation $p^{+}$and the deep derived relation $d^{+}$.

Third, observe that if $c(z)=x$ and $c\left(z^{\prime}\right)=x^{\prime}$, where $l(z)=l\left(z^{\prime}\right)$ and $\left(x^{\prime}, x\right) \in d$ and either $x^{\prime} \in D^{\prime}$ or $x \in D^{\prime}$, then $z \in U$ and $z^{\prime} \in U$. Moreover, if $p(z)$ or $p\left(z^{\prime}\right)$ is defined, then both are defined and both are in $U$. Hence, the derivation invariant holds with the subsystem $S_{U}$ for all three alternatives in definition 5 .

To understand the consequences of property 1 and theorem 3 , consider the example in figure 4. Suppose that we wish to construct a persistent representation of .T.Y and all the objects it deeply contains. We could simply represent the subsystem given by

$$
D^{\prime}=\{. T . Y, . T . Y . c 1, \cdots, . \text { T.Y.c4, .T.Y.c1.z, } \cdots, \text {,T.Y.c4.z }\} .
$$

This set has nine objects, and has lost the fact that the objects labeled $\mathbf{z}$ are all implied and the fact that the objects labeled ci are all instances of the same class. To fix this, we instead consider the subsystem given by

$$
U=\uparrow_{c}^{d^{+}}\left(D^{\prime}\right)=\{. T . Y, . T . Y . c 1, \cdots, . T . Y . c 4, . T . Y . c 1 . z, \cdots, \text { T.Y.c4.z, }, \text { T.c, }, \text { T.c.z }\} .
$$

This subsystem has more objects (eleven), but now four of those are implied, so the persistent representation is smaller. Moreover, we have preserved essential elements of the structure of the subsystem. Specifically, theorem 3 guarantees that this subsystem satisfies the derivation invariant. Property 1 guarantees that every implied object in the original system that is contained in the subsystem is also implied in the subsystem. Thus, it guarantees that we can leave out of any persistent representation of the subsystem any object that is implied in the original system. In this sense, it yields optimally compact representations.

In the software implementation of actor-oriented classes in Ptolemy II, these formal properties are extensively exploited to minimize the size of persistent representations and to support reasonable copy-and-paste behavior for objects. Of course, 
if an implied object is overridden in some way, then we may need to represent it anyway.

\section{CONCLUSION}

We have argued that actor-oriented design can benefit from abstraction and modularity mechanisms similar to what has been developed in object-oriented languages. We have given a formalism that provides a structure for classes and inheritance. There are a number of issues that have been left out, but that amount to fairly obvious extensions. For example, we have not specified how our mechanism interacts with a type checking and type systems. Our formalism permits subclasses to have additional input ports, yet such ports seem to prevent a subclass from being viewed as a subtype of the base class since extra input ports will never receive any data. A similar contravariance issue applies to the types of parameters and ports, which have not been discussed. This departs from current practice in OO languages, where subclasses are substitutable for superclasses. A policy that delivers substitutability, however, cannot be independent of the model of computation. Whether an additional port in a subclass impedes substitutability, for example, is a semantic question about the model of computation. The actor-oriented mechanisms we give here refer only to the abstract syntax (structure and valuation) of a model, and hence are valid for any AO model of computation.

Note that although our formalism could be applied to more conventional OO languages, it is not obvious that this would be useful. Languages such as $\mathrm{C}++$,

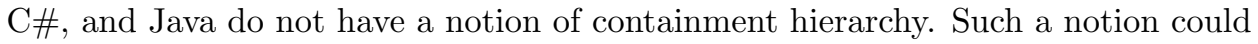
be superimposed using a design style, perhaps leading to useful design patterns. We leave this exploration for further work.

The mechanisms described here are implemented in Ptolemy II, available in open source form at http://ptolemy.org. They have been extensively tested with a comprehensive regression test suite and two years of practical user experience on models with up to tens of thousands of objects. Although the formalism is somewhat complex and intricate, the usage patterns are simple, and seem intuitive to users. Of course, it took decades for the details of object-oriented inheritance mechanisms to settle, so we can expect a similar evolution for actor-oriented inheritance mechanisms. But we humbly offer a well-founded starting point.

The benefits of any modularity mechanism, of course, must be weighed against the costs. Our mechanism adds surprisingly little run-time overhead. In particular, if the structure of a model does not change during execution of the model, then there is no run time overhead at all. The mechanisms proposed are not run-time mechanisms, and if models are compiled (code generated), then they have exactly zero impact on the cost of implementation. There is some run-time overhead if model structure is changed during execution and if valuations are changed. Note that allowing model structure to change during execution is not necessarily widely acknowledged to be a good idea (self-modifying code is generally not well thought of these days). Changing valuations can be useful, but even then, there are language design questions about whether valuations that are dynamically changing should be treated using the valuation mechanisms here or rather should be treated as dynamic data communicated between actors. In the latter case, there is again no 
run-time overhead due to the mechanisms we describe. For operations that are not performed at run time, we believe that as long as the complexity of the operations is no more than linear in the number of objects, then modest overhead is acceptable (modest meaning that it does not significantly alter the user experience). In our implementation in Ptolemy II, we noticed no degradation in user experience upon introducing these mechanisms.

\section{REFERENCES}

Agha, G. 1986. ACTORS: A Model of Concurrent Computation in Distributed Systems. The MIT Press Series in Artificial Intelligence. MIT Press, Cambridge, MA.

AGHA, G. 1990. Concurrent object-oriented programming. Communications of the ACM 33, 9, $125-140$.

Agha, G., Frolund, S., Kim, W., Panwar, R., Patterson, A., and Sturman, D. 1993. Abstraction and modularity mechanisms for concurrent computing. IEEE Parallel and Distributed Technology: Systems and Applications 1, 2, 3-14.

Agha, G. A., Mason, I. A., Smith, S. F., and Talcott, C. L. 1997. A foundation for actor computation. Journal of Functional Programming 7, 1, 1-72.

Arvind, Nikhil, R. S., Rosenband, D., and Dave, N. 2004. High-level synthesis: An essential ingredient for designing complex asics. In International Conference on Computer Aided Design (ICCAD). San Jose, CA.

Baccelli, F., Cohen, G., Olster, G. J., And Quadrat, J. P. 1992. Synchronization and Linearity, An Algebra for Discrete Event Systems. Wiley, New York.

Buesse, P., Claessen, K., Sheeran, M., And Singh, S. 1998. Lava: Hardware design in haskell. In International Conference on Functional Programming. 174-184.

Brooks, C., Lee, E. A., Liu, X., Neuendorffer, S., Zhao, Y., and Zheng, H. 2004. Heterogeneous concurrent modeling and design in Java. Tech. Rep. Technical Memorandum UCB/ERL M04/27, University of California. July 29.

Buck, J. T., Ha, S., Lee, E. A., And Messerschmitt, D. G. 1994. Ptolemy: A framework for simulating and prototyping heterogeneous systems. Int. Journal of Computer Simulation, special issue on "Simulation Software Development" 4, 155-182.

CRAIG, I. 2001. The Interpretation of Object-Oriented Programming Languages. Springer-Verlag.

Dennis, J. B. 1974. First version data flow procedure language. Tech. Rep. MAC TM61, MIT Laboratory for Computer Science.

Eker, J. And Janneck, J. W. 2003. Cal language report: Specification of the cal actor language. Tech. Rep. Technical Memorandum No. UCB/ERL M03/48, University of California, Berkeley, CA. December 1.

Eker, J., Janneck, J. W., Lee, E. A., Liu, J., Liu, X., Ludvig, J., Neuendorffer, S., Sachs, S., And Xiong, Y. 2003. Taming heterogeneity-the Ptolemy approach. Proceedings of the IEEE 91, 2, 127-144.

Esser, R. 1996. Ph.d. thesis. Ph.D. thesis, ETH.

Esser, R. AND JANNECK, J. W. 2001. A framework for defining domain-specific visual languages. In Workshop on Domain Specific Visual Languages, in conjunction with ACM Conference on Object-Oriented Programming, Systems, Languages and Applications OOPSLA-2001. Tampa Bay, Florida, USA.

Fritzson, P. 2003. Principles of Object-Oriented Modeling and Simulation with Modelica 2.1. Wiley.

Gamma, E., Helm, R., Johnson, R., And Vlissides, J. 1994. Design Patterns: Elements of Reusable Object-Oriented Software. Addison Wesley.

Goessler, G. and Sangiovanni-Vincentelli, A. 2002. Compositional modeling in metropolis. In Second International Workshop on Embedded Software (EMSOFT). Springer-Verlag, Grenoble, France.

Goessler, G. and Sifakis, J. 2005. Composition for component-based modeling. Science of Computer Programming 55.

ACM Journal Name, Vol. V, No. N, Month 20YY. 
Herrera, F. ANd Villar, E. 2006. A framework for embedded system specification under different models of computation in systemc. In Design Automation Conference (DAC). ACM, San Francisco.

HewitT, C. 1977. Viewing control structures as patterns of passing messages. Journal of Artifical Intelligence 8, 3, 323363.

Jackson, E. K. And Sztipanovits, J. 2006. Towards a formal foundation for domain specific modeling languages. In EMSOFT. ACM Press, Seoul, Korea, 53-62.

JAntsch, A. AND SAnder, I. 2005. Models of computation and languages for embedded system design. IEE Proceedings on Computers and Digital Techniques 152, 2, 114-129.

John Davis, I. 2000. Ph.d. thesis. Ph.D. thesis, UC Berkeley.

Johnson, S. C. 1994. Objecting to objects. In USENIX Winter 1994 Technical Conference Proceedings. San Francisco, California.

KARsaI, G. 1995. A configurable visual programming environment: A tool for domain-specific programming. IEEE Computer, 36-44.

Karsai, G., Maroti, M., Ldeczi, K., Gray, J., And Sztipanovits, J. 2003. Type hierarchies and composition in modeling and meta-modeling languages. IEEE Transactions on Control System Technology to appear.

Keutzer, K., Malik, S., Newton, A. R., Rabaey, J., and Sangiovanni-Vincentelli, A. 2000. System level design: Orthogonolization of concerns and platform-based design. IEEE Transactions on Computer-Aided Design of Integrated Circuits and Systems 19, 12.

Ledeczi, A., Bakay, A., Maroti, M., Volgyesi, P., Nordstrom, G., Sprinkle, J., and Karsai, G. 2001. Composing domain-specific design environments. IEEE Computer, 44-51.

LeE, E. A. 2006. The problem with threads. Computer 39, 5, 33-42.

Lee, E. A. And Neuendorffer, S. 2000. Moml - a modeling markup language in xml. Tech. Rep. UCB/ERL M00/12, UC Berkeley. March 14.

Lee, E. A., Neuendorffer, S., And Wirthlin, M. J. 2003. Actor-oriented design of embedded hardware and software systems. Journal of Circuits, Systems, and Computers 12, 3, 231-260.

Liao, S., Tuiang, S., And Gupta, R. 1997. An efficient implementation of reactivity for modeling hardware in the scenic design environment. In Design Automation Conference. ACM, Inc., Anaheim, CA.

Lynch, N., Segala, R., Vaandrager, F., and Weinberg, H. 1996. Hybrid I/O automata. In Hybrid Systems III, R. Alur, T. Henzinger, and E. Sontag, Eds. Vol. LNCS 1066. SpringerVerlag, 496-510.

Lynch, N. A. 1996. Distributed Algorithms. Morgan Kaufmann.

Mathaikutty, D. A., Patel, H. D., And Shukla, S. K. 2004. A functional programming framework of heterogeneous model of computation for system design. In Forum on Design and Specification Languages (FDL). Lille, France.

Patel, H. D. and Shukla, S. K. 2004. SystemC Kernel Extensions for Heterogeneous System Modelling. Kluwer.

Priess-Crampe, S. And Ribenboim, P. 1996. Generalized ultrametric spaces I. Abhandlungen aus dem Mathematischen Seminar der Universität Hamburg 66, 55-73.

RePpy, J. H. 1991. Cml: A higher-order concurrent language. SIGPLAN Notices 26, 6, 293-305.

SANDER, I. AND JANTSCH, A. 2004. System modeling and transformational design refinement in forsyde. IEEE Transactions on Computer-Aided Design of Circuits and Systems 23, 1, 17-32.

Selic, B., Gullekson, G., And Ward, P. 1994. Real-Time Object-Oriented Modeling. John Wiley and Sons, New York, NY.

Stewart, D. B., Volpe, R., And Khosla, P. 1997. Design of dynamically reconfigurable real-time software using port-based objects. IEEE Trans. on Software Engineering 23, 12, 759-776.

Strehl, K., Thiele, L., Gries, M., Ziegenbein, D., Ernst, R., And Teich, J. 2001. Funstatean internal design representation for codesign. IEEE Trans. on Very Large Scale Integration (VLSI) Systems 9, 4, 524-544.

Sutherland, I. E. 1963. Sketchpad - a man-machine graphical communication system. Tech. Rep. Technical Report 296, MIT Lincoln Laboratory. January. 
Sutherland, W. R. 1966. Ph.d. thesis. Ph.D. thesis, Mit.

Thies, W., Karczmarek, M., And Amarasinghe, S. 2002. Streamit: A language for streaming applications. In 11th International Conference on Compiler Construction. Vol. LNCS 2304. Springer-Verlag, Grenoble, France.

Wan, Z., Taha, W., And Hudak, P. 2001. Real-time FRP. ACM SIGPLAN Notices 36, 10, 146 -156 .

ACM Journal Name, Vol. V, No. N, Month 20YY. 